\title{
Localization Transition in Multilayered Disordered Systems
}

\author{
S.N. Evangelou ${ }^{1}$, Shi-Jie Xiong ${ }^{2}$, P. Markoš ${ }^{3}$ and D.E. Katsanos ${ }^{1}$ \\ ${ }^{1}$ Department of Physics, University of Ioannina, Ioannina 451 10, Greece \\ ${ }^{2}$ Department of Physics and National Laboratory of Solid State Microstructures, \\ Nanjing University, Nanjing 210008, China \\ 3 Institute of Physics, Slovak Acad. Sci, Dúbravská cesta 9, Bratislava 842 28, Slovakia
}

The Anderson delocalization-localization transition is studied in multilayered systems with randomly placed interlayer bonds of density $p$ and strength $t$. In the absence of diagonal disorder $(W=0)$, following an appropriate perturbation expansion, we estimate the mean free paths in the main directions and verify by scaling of the conductance that the states remain extended for any finite $p$, despite the interlayer disorder. In the presence of additional diagonal disorder $(W>0)$ we obtain an Anderson transition with critical disorder $W_{c}$ and localization length exponent $\nu$ independently of the direction. The critical conductance distribution $P_{c}(g)$ varies, however, for the parallel and the perpendicular directions. The results are discussed in connection to disordered anisotropic materials.

PACS numbers: 72.15.Rn, 71.30.+h, 74.25.Fy

\section{INTRODUCTION}

The understanding of the Anderson transition based on the scaling theory of localization [1] inspired many detailed numerical studies of disordered electronic systems [2]. The universality of the associated critical behavior was tested for various physical models, which include the crucial role of symmetry [3] and added magnetic field [ [ 1 . Universal critical transport properties are also expected in the presence of hopping matrix elements which are not the same in the various lattice directions, as it can be seen from computations for weakly coupled chains and coupled planes [5.6\%. It must be emphasized that many of the previous works on anisotropy include site diagonal isotropic disorder and involve anisotropy only in the hopping magnitudes. This kind of anisotropy remains for zero disorder and is manifested in the band structure. However, many realistic materials involve truly anisotropic disorder. For example, attempting to understand the high- $T_{c}$ cuprates within a non-interacting electron picture in the presence of disorder requires the explanation of the contrasting resistivities in the parallel and perpendicular directions [7 [10]. Anisotropic site randomness in a form resembling a random superlattice with lateral inhomogeneities gave anisotropic localization for anisotropy below a critical value, even for arbitrarily small disorder 11.

The in-plane resistivity for most of the layered high $-T_{c}$ materials exhibits metallic behavior, increasing linearly with temperature over a wide temperature range, while the perpendicular out-of-plane resistivity is very high at low temperatures and decreases rapidly as the temperature increases, reminiscent of semiconductors [12 15]. The contrasting behavior of the parallel and the perpendicular resistivities was observed in $\mathrm{Bi}_{2} \mathrm{Sr}_{2-x} \mathrm{La}_{x} \mathrm{CuO}_{y}$ far below $T_{c}$, down to the lowest experimental temperature 16]. In the underdoped $\mathrm{La}_{2-x} \mathrm{Sr}_{x} \mathrm{CuO}_{4}$ logarithmic divergencies of the corresponding resistivities accompanied by a nearly constant anisotropy ratio are, instead, observed suggesting an unusual three-dimensional $(3 D)$ insulator. The electronic transport in these materials is expected to arise from scattering in the "insulating" layer between the conducting $\mathrm{CuO}_{2}$ layers 12 15. On the other hand, in almost all high- $T_{c}$ cuprates doping impurities or oxygen vacancies occupy the insulating layers between the conducting "pure" $\mathrm{CuO}_{2}$ planes which implies interlayer disorder. Although the main aspects of transport in high- $T_{c}$ materials, such as the linear temperature dependence of the in-plane resistivity, are intimately connected with their strongly correlated nature, it is believed that anisotropic transport issues are, somehow, related to their layered structure.

We propose a strongly anisotropic multilayered structure (see Fig. 1) motivated by realistic anisotropic materials. This system involves truly anisotropic interlayer disorder, anisotropic hoppings and the usual isotropic site diagonal disorder. It can be regarded as a very simple model for the cuprates where the $\mathrm{CuO}_{2}$ planes are believed to be identical without superlattice-like disorder. Our aim is to study both parallel $(\|)$ and perpendicular $(\perp)$ transport addressing the following main questions: (1) does anisotropic localization occur (for example, localization in the layering direction and delocalization within the layers) for interlayer disorder only? (2) with additional isotropic diagonal disorder is the critical behavior independent of the direction as scaling theory predicts? Firstly, we compute the conductance in the case of interlayer disorder alone to check whether its localization behavior is the same in both directions. Secondly, in the presence of additional isotropic disorder of strength $W$ we obtain the critical disorder $W_{c}$ and the localization length critical exponent $\nu$ to see if they depend on the direction. We have also analyzed the statistical properties of the critical conductance distributions $P_{c}(g)$ 
and although we can conjecture that it is a unique singleparameter function the distribution for the logarithm of the critical $g$ in the "difficult" $\perp$ case resembles an insulator.

In Section II we proceed with the definition of the tight-binding Hamiltonian for the multilayered lattice structure. In Section III, we consider the system with only interlayer disorder and estimate the corresponding mean free paths in the two directions. Our results in the absence of isotropic diagonal disorder allow to conclude, in agreement with the scaling theory, that the states remain extended in both directions despite the strongly anisotropic interlayer disorder. However, the metallic conductance is very different for the $\perp$ case, being insulator-like. In Section IV we review the numerical methods for the computation of the conductance in cubic and long wire systems. We find singular behavior along the layering direction due to the missing bonds. In order to avoid this problem we have developed appropriate numerical algorithms based on transfer matrix and Green function methods. Finally, in Section V we discuss the conclusions of the present study also in connection to realistic systems.

\section{RANDOM MULTILAYER LATTICE}

We propose a simple $3 D$ anisotropic multilayered model which consists of parallel lattice planes randomly connected by interplane bonds as in Fig. 1 described by the Hamiltonian

$$
\begin{aligned}
H= & \sum_{\mathbf{m} l} \varepsilon_{\mathbf{m}, l}|\mathbf{m}, l><\mathbf{m}, l|+\sum_{\left\langle\mathbf{m}, \mathbf{m}^{\prime}\right\rangle, l}\left(\left|\mathbf{m}, l><\mathbf{m}^{\prime}, l\right|\right. \\
& \left.+t_{\mathbf{m}, l}^{\prime}|\mathbf{m}, l><\mathbf{m}, l+1|+\text { H. c. }\right),
\end{aligned}
$$

where $\mathbf{m}, \mathbf{m}^{\prime}$ denote the two-dimensional site indices in each layer and $l$ is the layer index. The first term in Eq. (1) describes diagonal (isotropic) disorder with the site matrix elements $\varepsilon$ chosen randomly from a box distribution within $[-W / 2, W / 2]$, the second term describes nearest-neighbor hopping of unit strength within the layers, which sets the energy unit, and the third term corresponds to interplane hoppings $t_{\mathbf{m}, l}^{\prime}=0$ or $t$, placed with probability $p$ at random layer positions $\mathbf{m}$. The interplane term obeys the binary distribution

$$
P\left(t_{\mathbf{m}, l}^{\prime}\right)=p \delta\left(t_{\mathbf{m}, l}^{\prime}, t\right)+(1-p) \delta\left(t_{\mathbf{m}, l}^{\prime}, 0\right) .
$$

The proposed structure has both anisotropic hoppings due to $t$ and anisotropic disorder due to $p$. The transport characteristics are obtained by calculating the conductance along the $\|$ and the $\perp$ directions.

The missing perpendicular bonds in the layering direction disturb particle migration even the presence of interlayer disorder alone. Unlike a naive expectation we find no critical point when $W=0$, for any $p \neq 0$. We show that the system is metallic independently of the direction, although the behavior of the conductance is very different in the two directions. In the presence of additional diagonal disorder, denoted by $W$, a critical disorder $W_{c}$ is obtained for various choices of the density $p$ and strength $t$. The critical point $W_{c}$ within finite size errors is found to be the same in both directions.

\section{MEAN FREE PATHS FOR INTERLAYER DISORDER}

We consider anisotropic disorder in the perpendicular layering direction represented by $p$ and $t$, due to the randomly placed bonds among consecutive layers, in the absence of diagonal disorder $W$. In this disordered anisotropic lattice one might expect transport to be hindered in the perpendicular direction. It is worth examining whether is present or not. In order to proceed we adopt a convenient layer-diagonal representation since for $W=0$ the $2 D$ layers are perfect planes and can be easily diagonalised. The eigenstates at the $l$-th layer $\left|\mathbf{k}_{\|}, l\right\rangle$ are labelled by the parallel momentum $\mathbf{k}_{\|}$and the Hamiltonian $H$ can be expressed in the convenient BlochWannier basis

$$
\left|\mathbf{k}_{\|}, l\right\rangle=\frac{1}{\sqrt{N_{\|}}} \sum_{\mathbf{m}} e^{i \mathbf{k}_{\|} \cdot \mathbf{m}}|\mathbf{m}, l\rangle,
$$

with parallel momentum $\mathbf{k}_{\|}$, the layer index $l$ and $\mathbf{m}$ summed over all $N_{\|}=L^{2}$ sites in every layer for a systen with $L^{3}$ sites. For $p=0$ the $2 D$ layers are perfect and $\mathbf{k}_{\|}$is a good quantum number. For $p=1$ the system reduces to a perfect $3 D$ lattice and both $\mathbf{k}_{\|}, k_{\perp}$ become good quantum numbers.

We consider the case of $p \neq 0,1$ where the translational symmetry in the plane directions is also broken and $\mathbf{k}_{\|}$ is no longer a good quantum number. In this mixed representation the Hamiltonian $H$ can be expressed as

$$
\begin{aligned}
H= & \sum_{\mathbf{k}_{\|}, l} \epsilon_{\|}\left(\mathbf{k}_{\|}\right)\left|\mathbf{k}_{\|}, l\right\rangle\left\langle\mathbf{k}_{\|}, l\right| \\
& +\sum_{l} \sum_{\mathbf{k}_{\|}, \mathbf{k}_{\|}^{\prime}}\left[t_{l, l+1}^{\mathbf{k}_{\|}, \mathbf{k}_{\|}^{\prime}}\left|\mathbf{k}_{\|}, l\right\rangle\left\langle\mathbf{k}_{\|}^{\prime}, l+1\right|+\text { H. c. }\right],
\end{aligned}
$$

with the parallel kinetic energy

$$
\epsilon_{\|}\left(\mathbf{k}_{\|}\right)=2 \cos \left(k_{x}\right)+2 \cos \left(k_{y}\right)
$$

and the hopping matrix element between neighboring planes

$$
t_{l, l+1}^{\mathbf{k}_{\|}, \mathbf{k}_{\|}^{\prime}}=\frac{1}{N_{\|}} \sum_{\mathbf{m}} e^{i\left(\mathbf{k}_{\|}^{\prime}-\mathbf{k}_{\|}\right) \cdot \mathbf{m}^{\prime}} t_{l, \mathbf{m}}
$$


In order to investigate the question of localization in the layering direction we define the retarded Green function

$$
G\left(\mathbf{k}_{\|}, l ; \mathbf{k}_{\|}^{\prime}, l^{\prime} ; t\right)=-i \theta(t)\left\langle\left[c_{\mathbf{k}_{\|}, l}(t), c_{\mathbf{k}_{\|}^{\prime}, l^{\prime}}^{\dagger}(0)\right]_{+}\right\rangle,
$$

where $c_{\mathbf{k}_{\|}, l}(t)$ is the time-dependent destruction operator of electron in the state $\left|\mathbf{k}_{\|}, l\right\rangle$. Its diagonal element $G\left(\mathbf{k}_{\|}, l ; \mathbf{k}_{\|}, l ; t\right)$ gives the probability for finding an electron on the layer $l$ with momentum $\mathbf{k}_{\|}$at time $t$, if initially it was on the same layer having the same momentum. The Fourier transformation of the diagonal Green function with respect to time is

$$
G\left(\mathbf{k}_{\|}, l ; \mathbf{k}_{\|}, l ; E\right)=\frac{1}{E-\Sigma\left(\mathbf{k}_{\|}, l, E\right)}
$$

with the self-energy $\Sigma_{\|}$written as 17]

$$
\Sigma\left(\mathbf{k}_{\|}, l, E\right)=\epsilon_{\|}\left(\mathbf{k}_{\|}\right)+\sum_{n=1}^{\infty} \sum_{j} T_{j}^{(n)} .
$$

The $n$-th order term is a sum over all paths $j$ of length $n$ starting and ending in the same state $\left|\mathbf{k}_{\|}, l\right\rangle$

$$
T_{j}^{(n)}=t_{l, l_{1}}\left(\mathbf{k}_{\|}, \mathbf{k}_{\| 1}\right) \prod_{i=1}^{n} \frac{t_{l_{i}, l_{i+1}}^{\mathbf{k}_{\| i} \mathbf{k}_{\| i+1}}}{E-\epsilon_{\|}\left(\mathbf{k}_{\| i}\right)}
$$

with $\mathbf{k}_{\| n+1}=\mathbf{k}_{\|}$and $l_{n+1}=l$. From Eq. (2) one has

$$
t_{l_{i}, l_{i+1}}^{\mathbf{k}_{\|}, \mathbf{k}_{\| i+1}}=\frac{t}{N_{\|}} \sum_{\mathbf{m} \in \mathcal{B}_{l_{i}, l_{i+1}}} e^{i\left(\mathbf{k}_{\| i}-\mathbf{k}_{\| i+1}\right) \cdot \mathbf{m}} \delta_{l_{i+1}, l_{i} \pm 1},
$$

with the sum for $\mathbf{m}$ over the set of sites $\mathcal{B}_{l_{i}, l_{i+1}}$ which have interplane bond connections. It is seen from Eq. (11) that the diagonal in momentum matrix elements $\left(\mathbf{k}_{\| i}=\right.$ $\mathbf{k}_{\| i+1}$ ) are exactly $p t$. For a given order $n$ if $E$ lies within the pure $2 D$ band $\epsilon_{\|}\left(\mathbf{k}_{\|}\right)$the most divergent term of Eq. (9) comes from the path which has intermediate states $\left|\mathbf{k}_{\| i}, l_{i}\right\rangle$ with $\epsilon_{\|}\left(\mathbf{k}_{\| i}\right) \sim E$ for all $i$. The corresponding term approaches $\left\{p t /\left[E-\epsilon_{\|}\left(\mathbf{k}_{\| i}\right)\right]\right\}^{n}$ which sequentially connects nearest neighbor plane states with the same $\mathbf{k}_{\| i}$. It can be also seen that momentum scattering is always accompanied by interlayer hopping since a change of $\mathbf{k}_{\|}$ leads to a change of the layer index.

The above analysis splits the Hamiltonian into two parts $H=H_{0}+H_{1}$. The "undisturbed" part $H_{0}$ represents a perfect anisotropic $3 D$ lattice with intralayer (interlayer) hopping $1(p t)$. The rest is a "random" Hamiltonian $H_{1}$ with only off-diagonal matrix elements expressed in the $H_{0}$-diagonal basis $|\mathbf{k}\rangle=\left|\mathbf{k}_{\|}, k_{z}\right\rangle$ of the form

$$
\begin{aligned}
H_{1}\left(\mathbf{k}, \mathbf{k}^{\prime}\right)= & \frac{t\left(e^{i k_{z}}+e^{-i k_{z}^{\prime}}\right)}{N} \sum_{l, \mathbf{m} \in \mathcal{B}_{l, l+1}} e^{i\left(\mathbf{k}_{\|}-\mathbf{k}_{\|}^{\prime}\right) \cdot \mathbf{m}+i\left(k_{z}-k_{z}^{\prime}\right) l}, \\
& \text { for } \mathbf{k}_{\|} \neq \mathbf{k}_{\|}^{\prime}, \\
H_{1}\left(\mathbf{k}, \mathbf{k}^{\prime}\right)= & 0, \text { for } \mathbf{k}_{\|}=\mathbf{k}_{\|}^{\prime},
\end{aligned}
$$

where $N$ is the total number of lattice sites and $k_{z}$ the perpendicular momentum.

It can be seen from Eq. (12) that the matrix elements of $H_{1}$ are complex numbers of average amplitude value modulo 1 plus a random phase. If the size of the system increases to infinity the phase exhausts all possible values in $[0,2 \pi]$ and the average should vanish. In this situation the scattering by the random configurations of the interplane bonds can be well described by perturbation theory with $\mathbf{k}$-space self-energy

$$
\Sigma(\mathbf{k}, E) \simeq \epsilon(\mathbf{k})+\sum_{\mathbf{k}^{\prime}} \frac{\left|H_{1}\left(\mathbf{k}, \mathbf{k}^{\prime}\right)\right|^{2}}{E-\epsilon\left(\mathbf{k}^{\prime}\right)-i 0^{+}} .
$$

The configuration average $\left\langle\operatorname{Im} \sum_{\mathbf{k}^{\prime}} \frac{\left|H_{1}\left(\mathbf{k}, \mathbf{k}^{\prime}\right)\right|^{2}}{E-\epsilon\left(\mathbf{k}^{\prime}\right)-i \Gamma}\right\rangle$ can be computed as a function of $p$ and the results fitted to a semicircular form as $\rho(E) t^{2} p(1-p)$, with $E$ within the $H_{0}$ band and $\rho(E)$ the corresponding density of states. This allows to estimate the lifetime of states $\tau \sim \frac{1}{\rho(E) t^{2} p(1-p)}$ and the corresponding mean free paths

$$
\begin{gathered}
\lambda_{\|}=\frac{\tau u_{\|}^{2}}{u} \simeq \frac{1}{\rho(E) t^{2} p(1-p) \sqrt{2+p^{2} t^{2}}}, \\
\lambda_{\perp}=\frac{\tau u_{\perp}^{2}}{u} \simeq \frac{p}{\rho(E)(1-p) \sqrt{2+p^{2} t^{2}}}
\end{gathered}
$$

for Fermi velocites $u_{\|(\perp)}$.

We observe that for small- $p$ the obtained mean free path in the parallel (perpendicular) direction is proportional to $1 / p(p)$. This implies that the scattering strength of the interlayer disorder increases with $p$ for parallel transport but decreases with $p$ for perpendicular transport. This intrinsically anisotropic situation seems in contradiction with the scaling theory of localization because $p \rightarrow 0$ might be thought to be in favor of localization in the perpendicular direction, while transport is the least affected giving extended states in the parallel direction. However, the application of the one-parameter scaling theory to this situation should give a common critical point in all directions so that transport in the perpendicular direction should be extended as well. This is, indeed, numerically confirmed in Chapter IV where we show that the scaling behavior of the conductance in finite cubic systems with interlayer disorder is in agreement with the scaling theory. It turns out that the interlayer disorder is not sufficient to localize the electrons, even in the "difficult" layering direction, without any additional diagonal disorder $W$. The statistical behavior of the conductance, however, is very different in the two directions. 


\section{NUMERICAL CALCULATION OF THE CONDUCTANCE}

\section{A. The cube}

The parallel (perpendicular) conductance $\frac{e^{2}}{h} g_{\|(\perp)}(L)$ can be obtained for a $L \times L \times L$ cubic system at the Fermi energy $E$ directly from the multichannel LandauerButtiker formula 18

$$
g_{\|(\perp)}(L)=\operatorname{Tr}\left(\mathbf{t}_{\|(\perp)}^{+} \mathbf{t}_{\|(\perp)}\right),
$$

where $\mathbf{t}_{\|(\perp)}$ is the transmission matrix for electronic propagation along the $\|(\perp)$ direction computed by transfer matrix techniques. Two perfect semi-infinite bars are attached to two opposite sides of this cube and hard-wall boundary conditions are used for the other sides. The number of independent channels for the incoming and outgoing leads is $L^{2}$ and the transmission matrix can be calculated from the amplitudes of transmitted waves in the outgoing leads by assigning, at a time, a unit incident wave amplitude for one channel in the incoming leads and zero for the rest. We can easily establish the recursion relations for the corresponding wave function coefficients along the parallel direction to calculate $g_{\|}$. In the $\perp$ direction computing the matrix $\mathbf{t}_{\perp}$ is not possible since the recursion relations are singular due to the presence of zero hoppings for the missing interplane bonds. To overcome this difficulty we set up the recursion relations along the direction parallel to the planes, perpendicular to the leads, but with the reflection and transmission coefficients in the channels as the unknown variables. We can solve these recursion relations with hard wall boundary conditions perpendicular to the leads. By this method we obtain the perpendicular transmission matrix $\mathbf{t}_{\perp}$ avoiding the singularities due to missing bonds. This is a convenient tool to consider propagation in the layering $\perp$ direction by a transfer matrix product only along the easy $\|$ direction. In order to suppress fluctuations we have taken averages over up to a 5000 random cubic configurations in each case [19].

\section{B. The wire}

We can also compute the parallel (perpendicular) dimensionless conductance $g_{\|(\perp)}(M)$ for a quasi-onedimensional $M \times M \times L$ geometry, via Green function methods 20]. In the parallel direction $\mathbf{m}=x, y$ the Hamiltonian of the $M \times M$ slice is incorporated into the transfer matrix

$$
T_{x}=\left(\begin{array}{cc}
V_{x, x+1}^{-1} & 0 \\
0 & 1
\end{array}\right)\left(\begin{array}{cc}
E-H_{x} & -1 \\
1 & 0
\end{array}\right)\left(\begin{array}{cc}
1 & 0 \\
0 & V_{x-1, x}
\end{array}\right),
$$

where the matrix $V_{x, x+1}$ has unit elements. For large length $L$ in the $x$-direction the product

$$
T=\prod_{x=1}^{L} T_{x}
$$

has eigenvalues $\exp \gamma_{i} L$ and Lyapunov exponents $\gamma_{i}, i=$ $1,2, \ldots, M^{2}$. The smallest positive Lyapunov exponent $\gamma_{1}$ determines the scaling parameter $\Lambda_{\|}$, via

$$
\Lambda_{\|}^{-1}=M \gamma_{1}=\left(\frac{\xi_{M}}{M}\right)^{-1}
$$

with $\xi_{M}=\frac{1}{\gamma_{1}}$ the largest localization length, which is of interest for finite-size scaling studies when the width $M^{2}$ of the slice is varied. We obtain the critical value $\Lambda_{c \|}$ at the point where $\Lambda_{\|}$becomes independent of $M$ (see Table 1). In order to ensure accuracy of about $1 \%$ for $\Lambda_{\|}$the length of the studied system is more than about 200000. We find that this length near the critical point varies as $\propto \Lambda^{-1}$. In the case of very strong anisotropy we were unable to obtain this accuracy for all $M$ and $W$.

This method cannot be used in the $\perp$-direction. The reason is, again, the zero elements of the hopping matrix $V_{l, l+1}$ with probability $1-p$ so that the inverse matrix $V_{l, l+1}^{-1}$ which enters (17) becomes singular. In order to avoid this problem we can alternatively use the Green function $G(E)=(E-H)^{-1}$ by applying the iterative scheme of 20] based on the two equations

$$
G_{1, l+1}^{(l+1)}=G_{1, l}^{(l)} V_{l, l+1} G_{l+1, l+1}^{(l+1)}
$$

and

$$
G_{l+1, l+1}^{(l+1)}=\left[E-H_{l+1}-V_{l+1, l} G_{l, l}^{(l)} V_{l, l+1}\right]^{-1} .
$$

The Hamiltonian $H_{l}$ represents the $l$ th $M \times M$ layer, $V_{l, l+1}$ the hopping between layers $l, l+1$ and $G_{l_{1}, l_{2}}^{(l)}$ is the Green function of the system with length $l$ between layers $l_{1}$ and $l_{2}$. The diagonal matrix $V_{l, l+1}$ of the order of $M^{2}$ has zeros with probability $1-p$ so that $V_{l, l+1}^{-1}$ becomes singular. The main advantage of the formulae $(20,21)$ is that they do not contain the inverse of $V_{l, l+1}$. The disadvantage is the necessity to invert a matrix of order $M^{2}$ [Eq. (21)] at each iteration step. In this case we restrict the number of iterations to $L \leq 40000$ and the corresponding peprendicular scaling parameter is defined as

$$
\Lambda_{\perp}^{-1}=\frac{M}{L} \log \operatorname{Tr} G_{1 L}^{L}=\left(\frac{\xi_{M}}{M}\right)^{-1}
$$

The critical disorder $W_{c}$ and the critical exponent $\nu$ are computed in both directions from the numerical data of $\Lambda(M, W)$. The usual linearization near the critical point

$$
\log \Lambda(M, W)=\alpha_{M}+\beta_{M} \log W,
$$


and the independence of $\Lambda$ on $M$ at the critical point gives $W_{c}$ from the slope of the linear dependence

$$
\alpha_{M}=-\log W_{c} \times \beta_{M}+\text { const. }
$$

The obtained critical $\Lambda_{c \|}$ and $\Lambda_{c \perp}$ are different from the value $\Lambda_{c}$ of the corresponding isotropic system (see however Eq. (28) below). The critical exponent $\nu$ is determined from the coefficients $\beta_{M}$ via

$$
\nu=-\frac{\log M}{\log \beta_{M}} .
$$

We have also repeated the computations for cubic systems and although the finite size effects became more pronounced the obtained critical values differ very little from those obtained for long wires.

In agreement with the $W=0$ case it is reasonable to suppose that the form of $P_{c}(g)$ also depends on the direction. For the $\|$ direction the conductance is calculated from the formula

$$
g(M)=\sum_{i=1}^{M^{2}} \frac{2}{\cosh ^{2}\left(z_{i} / 2\right)}
$$

where the $z_{i}$ 's are the logarithms of the $i$ th eigenvalues of the matrix $\mathbf{t}^{\dagger} \mathbf{t}$ [21] and in the limit $L>>M$ converge to $2 M \gamma_{i}$. In the $\perp$ direction the singular behavior of the matrix $V_{l, l+1}$ does not permit to use the formula (26). To calculate $\log g_{\perp}$ in this case we use the fact that $\Lambda_{c \perp}<<$ 1 for most of the critical points discussed below. Then, the critical conductance from $z_{1}=2 / \Lambda_{c}$ is very small and can be estimated from the contribution of the first channel via

$$
\log g_{\perp}=\operatorname{Tr} G_{1, L}(E+i \eta) G_{L, 1}(E-i \eta),
$$

with the imaginary part of the energy $\eta$ given by the ratio of the bandwidth over the mean level spacing [22].

\section{RESULTS}

$$
\text { A. } W=0
$$

Fig. 2 shows the scaling behavior of $g_{\|(\perp)}(L)$ for the $L^{3}$-site cubic system for various bond densities $p$ and anisotropic interplane coupling $t=0.3$, in the absence of diagonal disorder $W$. The parallel conductance is shown to increase ballistically $\left(\sim L^{2}\right)$ for small $L$ and linearly for higher $L$. In the large size $(L \rightarrow \infty)$ limit the corresponding scaling function $\beta(L)=d \ln g / d \ln L$ becomes positive for $g_{\|(\perp)}$, which implies extended states in both directions for any $p$, in agreement with the scaling theory which predicts a common critical point in any direction. However, the obtained transport behavior is essentially different in the two directions. In Fig. 3 we show the energy dependence of the conductance for $p=0.5$ and $t=0.3$ where the ratio of the two conductances is close to the estimate $g_{\|} / g_{\perp} \approx\left(t_{\|} / t_{\perp}\right)^{2}$ for $t_{\|}=1$ and $t_{\perp}=p t=0.15$ [6]. A key finding from Fig. 2 is a rather smooth $g_{\|}(E)$ while $g_{\perp}(E)$ displays violent oscillations as a function of $E$. The dips in $g_{\perp}(E)$ can be regarded as due to "minigaps" in the perpendicular direction which might have effect similar to a semiconductor, leading to insulating kind of behavior for the out-of-plane conductivity when the Fermi energy is varied.

$$
\text { B. } W>0
$$

The $W$-dependence of $\Lambda(M, W)$ for different $M$ 's and various parameters $p, t$ is presented in Figs 4., 5., 6., 7 . The corresponding critical points are calculated by the described fitting procedure. The results are listed in Table 1 and the data are very reliable for the $\|$ direction. In the $\perp$ direction they are much harder to analyze due to finite-size effects. For example, in case D (Fig. 7) the obtained $M$-dependence of $\Lambda_{\perp}(M)$ is not monotonic and for $W=5$ we find $\Lambda(M)$ which decreases with $M$ for small system sizes, imitating insulating behavior. However, for larger $M>12$ the values of $\Lambda(M)$ begin to increase and the correct scaling is restored. This $M$ dependence is caused by a second irrelevant scaling parameter from the relation $\Lambda=a M^{1 / \nu}+b M^{\beta}$ with $\beta<0$. The correct estimation of the critical parameters in the $\perp$

\begin{tabular}{|c|c|c|c|c|c|c|c|c|}
\hline & $p$ & $t$ & $W_{c}$ & $\Lambda_{c}$ & $\nu$ & $M$ & $\langle g\rangle$ & $\operatorname{var} g$ \\
\hline A & 0.6 & 1.0 & $14.47 \pm 1.10$ & 0.676 & $1.619 \pm 0.14$ & $10-16$ & 0.59 & 0.16 \\
\hline $\mathrm{B}$ & 0.6 & 0.3 & $10.48 \pm 0.58$ & 0.933 & $1.666 \pm 0.10$ & $6-14$ & 1.5 & 0.4 \\
\hline $\mathrm{C}$ & 0.1 & 0.3 & $7.93 \pm 0.24$ & 1.218 & $1.514 \pm 0.06$ & $12-18$ & 2.6 & 0.7 \\
\hline $\mathrm{D}$ & 0.6 & 0.1 & $8.05 \pm 0.45$ & 1.174 & $1.790 \pm 0.14$ & $6-16$ & 2.6 & 0.7 \\
\hline \multicolumn{9}{|c|}{ 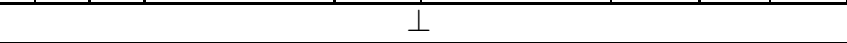 } \\
\hline $\mathrm{A}$ & 0.6 & 1.0 & $14.30 \pm 1.30$ & 0.437 & $1.546 \pm 0.16$ & $6-14$ & & \\
\hline $\mathrm{B}$ & 0.6 & 0.3 & $10.20 \pm 0.67$ & 0.189 & $1.308 \pm 0.21$ & $8-18$ & & \\
\hline $\mathrm{C}$ & 0.1 & 0.3 & $7.18 \pm 0.74$ & 0.089 & - & $12-20$ & & \\
\hline $\mathrm{D}$ & 0.6 & 0.1 & $6.80 \pm 1.94$ & 0.100 & - & $14-18$ & & \\
\hline
\end{tabular}
direction requires either numerical data for larger $M$ or possibly more sophisticated fits [23]. Although for strong anisotropy we could not obtain accurately the critical parameters in the $\perp$ direction we check that our results converge to those of the $\|$ direction when $M$ grows.

TABLE I. Review of various critical parameters with the mean conductance $\langle g\rangle$ and its variance var $g=\left\langle g^{2}\right\rangle-\langle g\rangle^{2}$. The obtained values confirm that the critical disorder is very close for $\|$ and $\perp$ transport, in agreement with the one-parameter scaling theory. We could not calculate the critical exponent $\nu$ in the $\perp$ direction for the cases C, D. 
In this direction the critical region is very narrow and we could neither calculate the critical exponent, since larger system sizes are required. Nevertheless, the scaling analysis for A and B gives satisfactory results in both directions which confirm the equal $W_{c}$ and $\nu$. Moreover, we find the quantity

$$
\Lambda_{c}=\left[\Lambda_{c \|}^{2} \times \Lambda_{c \perp}\right]^{1 / 3}
$$

which gives the critical value of the corresponding isotropic model [6].

Fig. 8(a), (b) presents the probability distribution of the critical conductance $P_{c}\left(g_{\|}\right)$in the parallel direction. The distribution is shown to be size-independent but depends on the various critical points. For $\mathrm{C}$ and $\mathrm{D}$ the obtained $P_{c}\left(g_{\|}\right)$and $\Lambda_{c \|}$ are the same. This indicates that $P_{c}\left(g_{\|}\right)$is determined only by $\Lambda_{c \|}$ [21]. The numerical data for the mean and variance of the conductance are also unique functions of $\Lambda_{c \|}$ (see Table 1) supporting this conjecture. In the limit $t \rightarrow 0$ the critical distribution in the parallel direction is expected to converge to a Gaussian. However, the spectrum of the obtained higher Lyapunov exponents shows square-root behavior similar to isotropic $3 D$ disordered systems [22]. To display the dramatic differences in parallel and perpendicular transport, we also present the critical distribution of $\log g_{\perp}$, calculated for the perpendicular direction for the critical case C. This distribution has all the features of the localized regime since it is log-normal with

$$
\operatorname{var} \log g_{\perp} \approx-\left\langle\log g_{\perp}\right\rangle .
$$

The important difference with the insulating regime is the fact that $P_{c}\left(\log g_{\perp}\right)$ remains system-size invariant. The relation of Eq. (29) is also valid for the critical points $\mathrm{B}$ and $\mathrm{D}$ where in the $\perp$ direction $\left\langle g_{\perp}\right\rangle<<1$.

\section{DISCUSSION}

The random topological multilayered structure studied may be regarded as a first step towards an explanation, via non-interacting electrons, for properties of strongly anisotropic materials. For example, in the case of $W=0$ the $2 D$ layers are perfect and the disorder represented by $p$ can be due to impurities or oxygen vacancies in the insulating layer among the $2 D$ planes of the cuprates. The electrons propagating in the perpendicular direction of this system shall encounter anisotropy in the disorder due to the random interplane links in addition to the value of $t$ which can be different to that of the parallel direction. However, the distribution of the critical conductance $P_{c}(g)$ depends both on the choice of the parameters and the direction where the electron moves.

The considered anisotropic structure exhibits rather strange transport properties on a given scale, expressed in the dramatic differences of the critical conductance in the parallel and perpendicular directions. In the perpendicular direction the conductance distribution slightly bellow the critical point is log-normal resembling the statistical properties typical of an insulator. A similar statistical "anomaly" has been described in [20]. However, a strong difference to a "true" insulating regime exists since the conductance still grows with the size. In the large size limit the corresponding distribution reaches a Gaussian. An analogous discussion holds for the parallel direction slightly above the critical point. The main criterion for the specification of the critical regime is the size dependence of the conductance $\langle g\rangle$ (or $\langle\log g\rangle$ ).

The proposed model for $W=0$ may have some relation to the strongly anisotropic transport proprties of high- $T_{c}$ cuprates. In these materials, as temperature increases, the inelastic scattering due to phonons, spin waves or other excitations within the $\mathrm{CuO}$ planes, can cause a decrease of the inelastic scattering length $l_{i n}$. If the temperature is so high that $l_{i n}$ becomes smaller than mean free path the transverse conductivity is metallic. Experiments for $\mathrm{Bi}_{2} \mathrm{Sr}_{2} \mathrm{CaCu}_{2} \mathrm{O}_{8}$ and underdoped $\mathrm{La}_{2-x} \mathrm{Sr}_{x} \mathrm{CuO}_{4}, \mathrm{YBa}_{2} \mathrm{Cu}_{3} \mathrm{O}_{6+x}$ give out-ofplane resistivity which has a semiconductor-like temperature dependence at low temperatures (high at small$T$ with a rapid decrease by increasing $T$ ) and a linearin- $T$ behavior at high temperatures. The characteristic crossover temperature between the two regimes $T^{*}$ decreases by increasing the doping in $\mathrm{La}_{2-x} \mathrm{Sr}_{x} \mathrm{CuO}_{4}$ and $\mathrm{YBa}_{2} \mathrm{Cu}_{3} \mathrm{O}_{6+x}$ [7] 10]. We notice that if we relate the bond density $p$ with the doping density of the high- $T_{c}$ materials the obtained $p$-dependence of the perpendicular mean free path can be used to explain qualitatively the reported behavior. It must be pointed out that the relation between the cuprate doping density and the bond density $p$ is natural, since an increase in the number of the doping impurities or the oxygen atoms in the layer between two $\mathrm{CuO}_{2}$ planes increases the number of hopping paths between the two planes. As $T^{*}$ decreases further (below $T_{c}$ ) the out-of-plane normal-state resistivity also becomes metallic, which has been observed in highquality single crystals of $\mathrm{YBA}_{2} \mathrm{Cu}_{3} \mathrm{O}_{7}$ and other high- $T_{c}$ cuprates corresponding to the absence of disorder $(p \approx 1$ with $W=0$ in the proposed model) with almost infinite perpendicular mean free path. This effect occurs only in the perpendicular direction since the parallel mean free path is always much longer and inelasting scattering becomes dominant.

In summary, we have introduced a simple layered lattice model with anisotropic disorder described by the interplane bond density $p$, in addition to the usual anisotropic band structure expressed via the interplane hopping $t$. In the absence of diagonal disorder we show extended states in both directions but the obtained mean free path and the conductance in the $\perp$ direction is much 
smaller than in the $\|$ direction. Moreover, $g_{\perp}$ fluctuates strongly as a function of energy, which leads to an insulator-like temperature dependence of the conductivity in the $\perp$ direction. In the presence of additional diagonal disorder of strength $W$ we have shown that the critical disorder and the critical exponent $\nu$ do not depend on the transport direction. The obtained data for the localization exponent $\nu$ agree with recent accurate estimates for the isotropic model 23] and confirm the universality at the metal-insulator transition. The obtained critical conductance distribution $P_{c}(g)$ although independent on the system size depends strongly on the parameters and the direction of transport.

\section{Acknowledgments}

This work was supported in part by a TMR network. SNE and SJX thank a Chino-Greek grant and PM the Slovak Grant Agency and NATO. We also like to thank Professors Xing and Economou for many useful discussions.

[1] E. Abrahams, P.W. Anderson, D.C. Licciardello and T.V. Ramakrishnan, Phys. Rev. Lett. 42, 673 (1979).

[2] For a review, see B. Kramer and A. MacKinnon, Rep. Prog. Phys. 56, 1469 (1993).

[3] S.N. Evangelou and T.A.L. Ziman, J. Phys. C: 20, L235 (1987); T. Ando, Phys. Rev. B 40, 5325 (1989); P. Markoš, J. Phys. I France 4, 551 (1994); Europhys. Lett. 26, 431 (1994).

[4] S. Das Sarma, in Perspectives in Quantum Hall Effects, edited by S. Das Sarma and Aron Pinczuk, (John Wiley and Sons, Inc., New York, 1997).

[5] N.A. Panagiotides, S.N. Evangelou, G. Theodorou, Phys. Rev. B 49, 14122 (1994).

[6] I. Zambetaki, Qiming Li, E. N. Economou, and C. M. Soukoulis, Phys. Rev. Lett. 76, 3614 (1996).

[7] For reviews, see Y. Iye, in Physical Properties of High Temperature Superconductors III, edited by D.M. Ginsberg, (World Scientific, Singapore, 1991), S.L. Cooper and K.E. Gray, in Physical Properties of High Temperature Superconductors IV, edited by D.M. Ginsberg, (World Scientific, Singapore, 1994).

[8] A. G. Rojo and K. Levin, Phys. Rev. B 48, 16861 (1993).

[9] A. A. Abrikosov, Phys. Rev. B 50, 1415 (1994).

[10] Y. Zha, S. L. Cooper, and D. Pines (unpublished).

[11] W. Xue, P. Sheng, Q.J. Chu, and Z.Q. Zhang, Phys. Rev. B 63, 2837 (1989); ibid. Phys. Rev. B 42, 4613 (1990); Q.J. Chu, and Z.Q. Zhang, ibid. 48, 10761 (1993).

[12] See the review, B. Batlogg, in High Temperature Superconductivity: The Los Alamos Symposium, edited by K. Bedell et al (Addison-Wesley, MA, 1990), p37.

[13] L. Forro, V. Ilakovac, B. Keszei, Phys. Rev. B 41, 9551 (1990).

[14] K. Takenaka, K. Mizuhashi, H. Takagi, and S. Uchida, Phys. Rev. B 50, 6534 (1994).
[15] Y. Nakamura and S. Uchida, Phys. Rev. B 47, 8369 (1993).

[16] Y. Ando, G.S. Boedinger, A. Passner, N.L. Wang, C. Geibel, and F. Steglich, Phys. Rev. Lett. 77, 2065 (1996) and references therein.

[17] D.J. Thouless, J.Phys. C 3, 1559 (1970); D.C. Licciardello and E.N. Economou, Phys. Rev. B 11, 3697 (1975).

[18] M. Buttiker, Y. Imry, R. Landauer, and S. Pinhas, Phys. Rev. B 31, 6207 (1985); E. N. Economou and C. M. Soukoulis, Phys. Rev. Lett. 46, 618 (1981); D. S. Fisher and P. A. Lee, Phys. Rev. B 23, 685 (1981).

[19] We have also calculated by exact diagonalization of the $L \times L \times L$ Hamiltonian the anisotropic inverse participation ratios defined by

$$
r_{\|}=\sum_{l}\left(\sum_{\mathbf{m}}\left|c_{\mathbf{m}, l}\right|^{2}\right)^{2}, \quad r_{\perp}=\sum_{m_{x}}\left(\sum_{l, m_{y}}\left|c_{\mathbf{m}, l}\right|^{2}\right)^{2}
$$

for the parallel and the perpendicular directions. The obtained localization length estimates are $\xi_{\|}=r_{\|}^{-1}$ and $\xi_{\perp}=r_{\perp}^{-1}$. We find $\xi_{\|}$always equal to the chosen system size $L$ while $\xi_{\perp}$ is much smaller.

[20] A. MacKinnon, B. Kramer, Phys. Rev. Lett. 47, 1546 (1981); A. MacKinnon, J. Phys.: Condens. Matter 13, L1031 (1980).

[21] P. Markoš, Phys. Rev. Lett. 82, 588 (1999).

[22] A. MacKinnon, Z. Phys. B 59, 385 (1985).

[23] K. Slevin and T. Ohtsuki, Phys. Rev. Lett. 82382 (1999) 


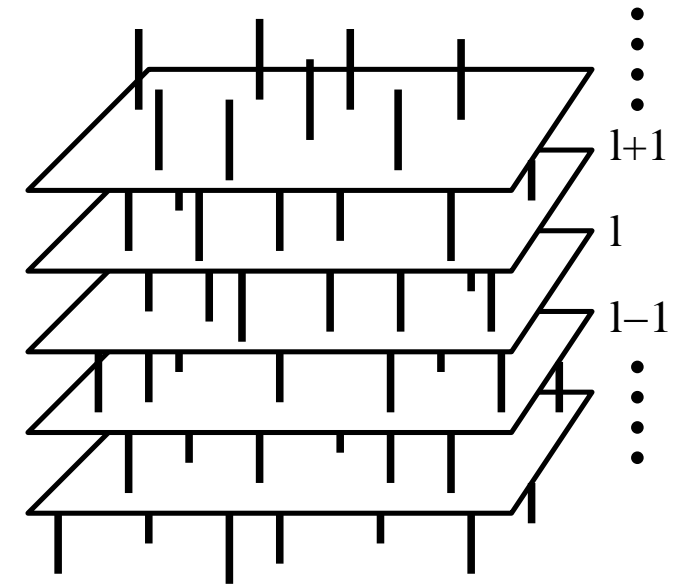

FIG. 1. A picture of the multilayered structure which consists of $2 D$ square lattices $l$ (layers) connected by perpendicular bonds of strength $t$ placed at random positions with probability $p$.

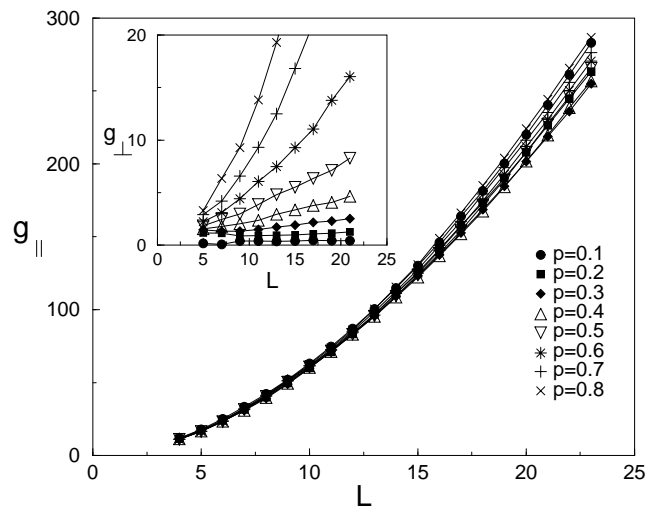

FIG. 2. The $g_{\|}$as a function of the linear system size $L$ for a cubic $L \times L \times L$ system of parallel planes with $W=0$ and randomly placed interplane bonds of density $p$ with strength $t=0.3$. In the inset $g_{\perp}$ for the same system exhibits similar behavior but much smaller values.

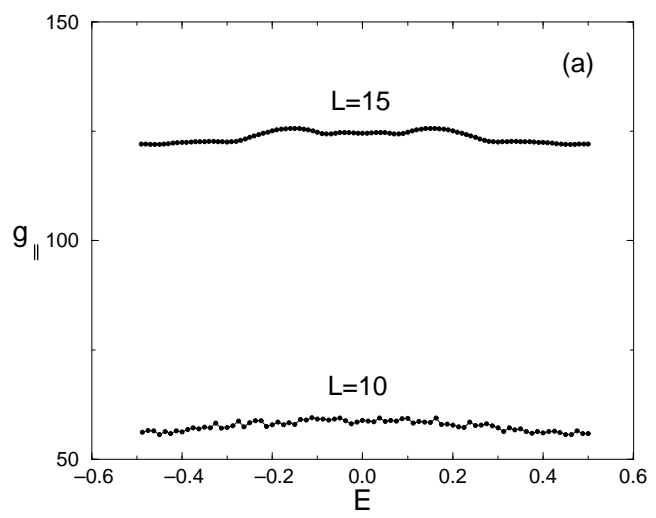

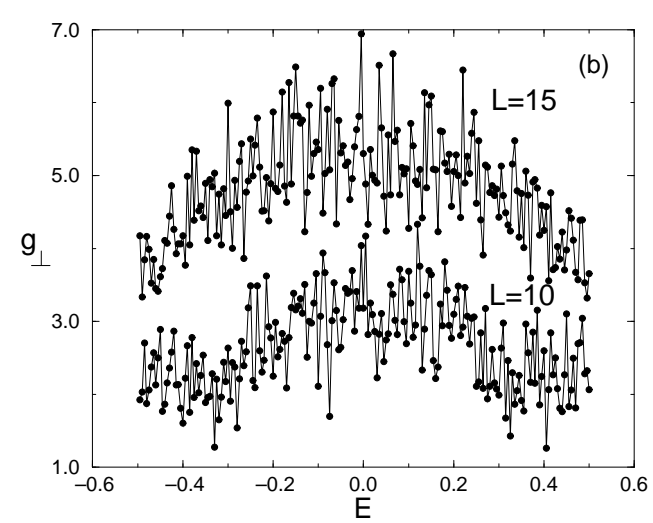

FIG. 3. (a) The energy-dependent $g_{\|}$for a cubic layered system with $L=10,15$ and $W=0, t=0.3, p=0.5$. (b) The $g_{\perp}$ is much smaller and exhibits violent oscillations as a function of the energy E.
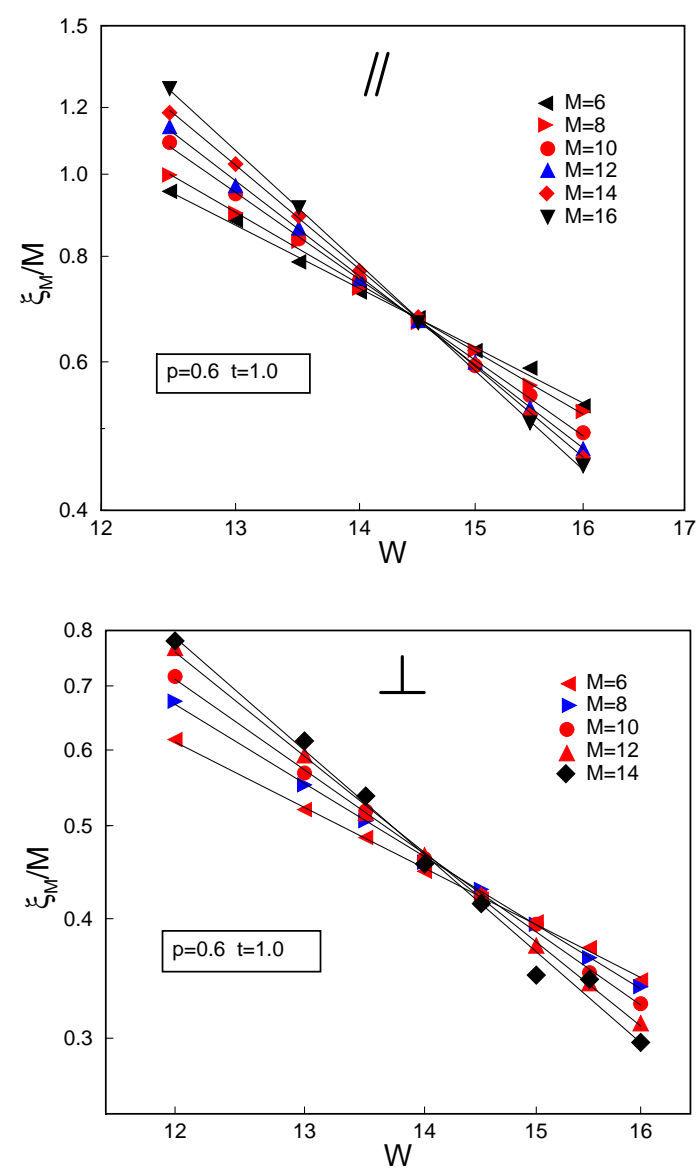

FIG. 4. A: The behavior of the scaled localization length $\xi_{M} / M$ for the parallel and perpendicular direction in the $M \times M \times L$ system with $p=0.6$ and $t=0.1$ as a function of $W$. The critical point is located at $W_{c} \approx 14.47$ in the $\|$ direction and $W_{c} \approx 14.30$ in the $\perp$ direction. 

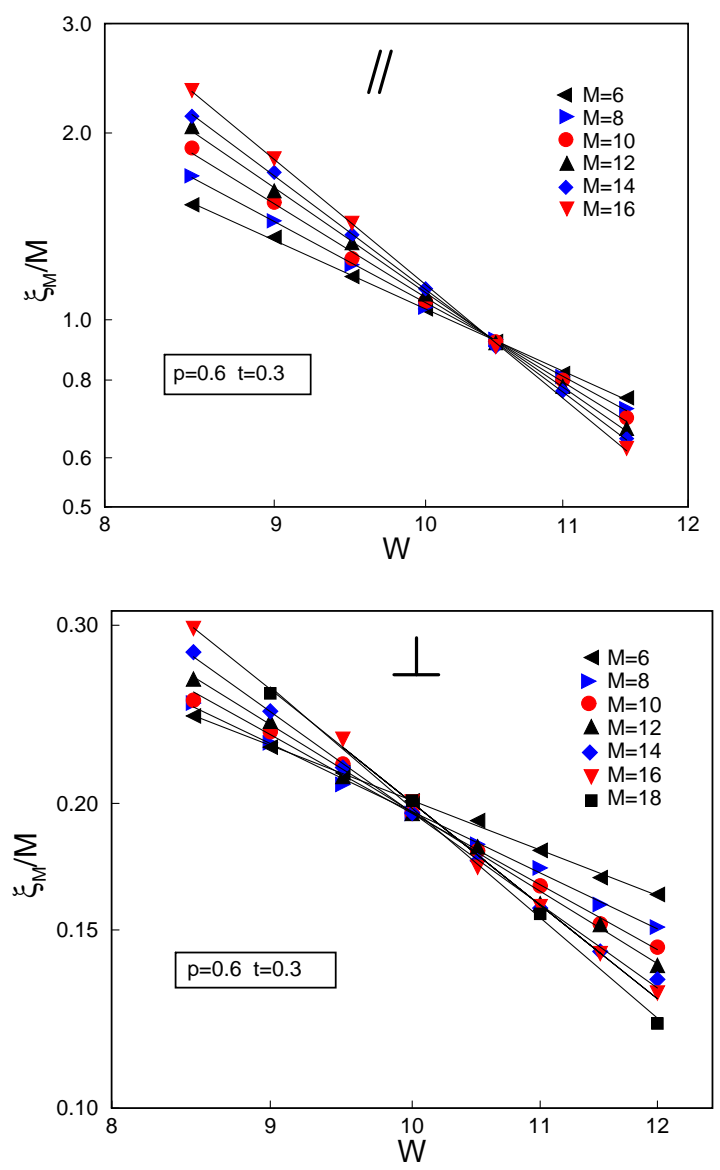

FIG. 5. B: The behavior of the scaled localization length $\xi_{M} / M$ for the parallel and perpendicular direction in the $M \times M \times L$ system with $p=0.6$ and $t=0.3$ as a function of $W$. The critical point is located at $W_{c} \approx 10.48$ in the $\|$ and $W_{c} \approx 10.20$ in the $\perp$ direction.

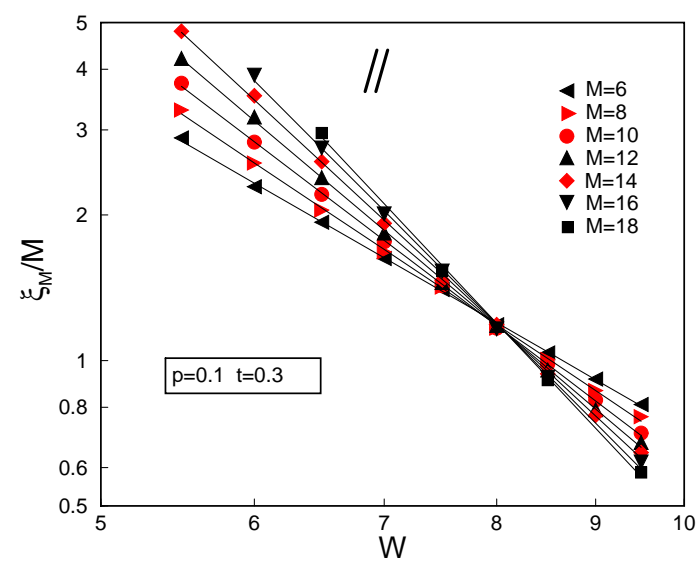

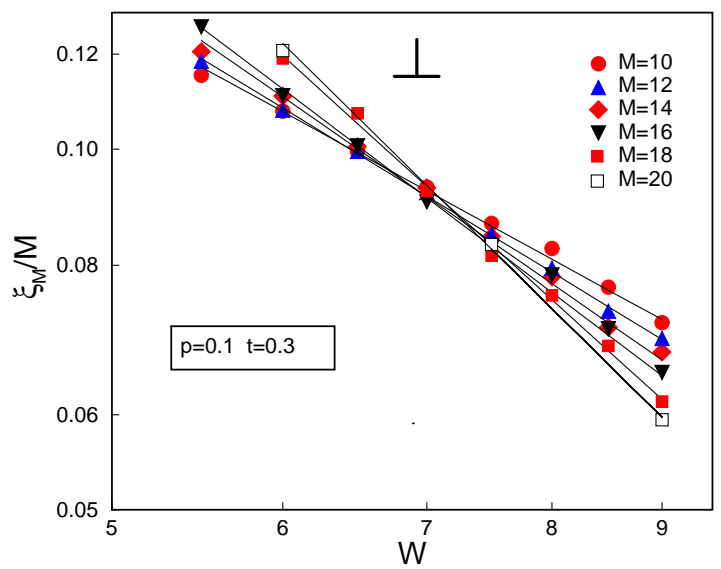

FIG. 6. C: The behavior of the scaled localization length $\xi_{M} / M$ for the parallel and perpendicular direction in the $M \times M \times L$ system for $p=0.1$ and $t=0.3$ as a function of $W$. The critical point is displayed at $W_{c} \approx 7.93$ in the $\|$ and $W_{c} \approx 7.18$ in the $\perp$ direction.
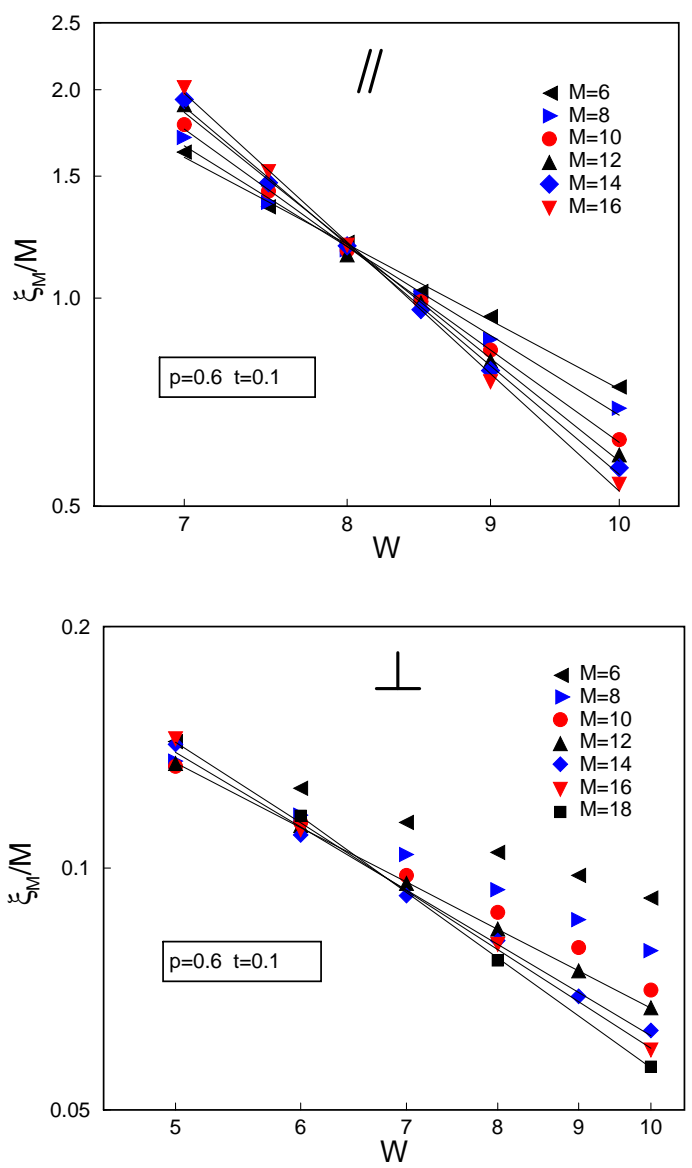

FIG. 7. D: The behavior of the scaled localization length $\xi_{M} / M$ for the $\|$ and $\perp$ direction in the $M \times M \times L$ system with $p=0.6$ and $t=0.1$ as a function of $W$. The critical point is located in $W_{c} \approx 8.05$ in the $\|$ and $W_{c} \approx 6.80$ in the $\perp$ direction. It is seen that the data for smaller $M=6,8$ fail to cross at the same point indicating "insulating" behavior. 

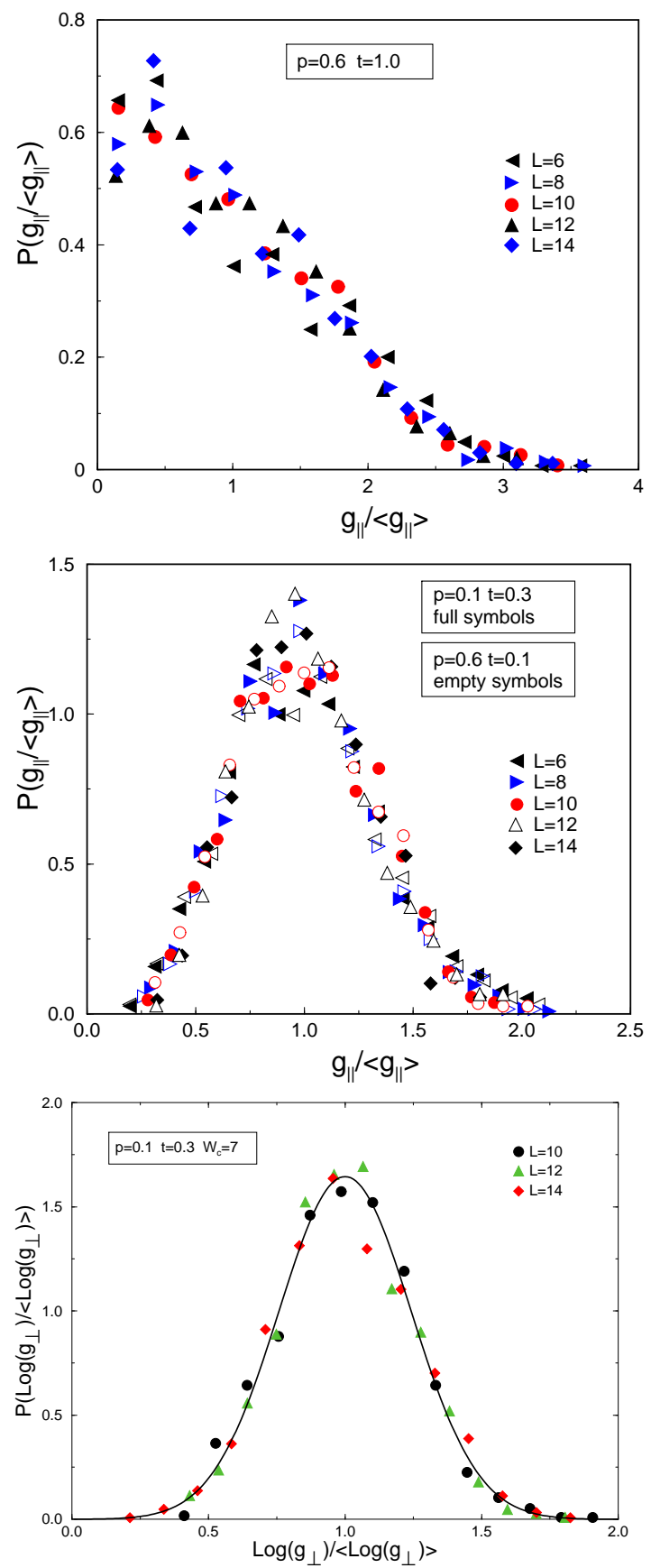

FIG. 8. (a). The critical distribution of the parallel conductance in the case A with $\left\langle g_{\|}>\approx 0.59, \operatorname{var}\left(g_{\|}\right)=0.16\right.$. (b). The same as in (a) for the critical points $\mathrm{C}$ (full symbols) and $\mathrm{D}$ (open symbols) with $\left\langle g_{\|}>\approx 2.6\right.$, var $\left(g_{\|}\right)=0.7$. The C,D have the same $\Lambda_{c}$ and the same critical distribution (see Table 1). (c). The critical distribution of $P\left(\log g_{\perp}\right)$ in the perpendicular direction for case $\mathrm{C}$ is also shown for comparison. 